\title{
Total brain, cortical, and white matter volumes in children previously treated with glucocorticoids
}

\author{
Sara K. Holm ${ }^{1,2}$, Kathrine S. Madsen ${ }^{2,3}$, Martin Vestergaard ${ }^{2}$, Olaf B. Paulson ${ }^{2,4}$, Peter Uldall ${ }^{1}$, Hartwig R. Siebner ${ }^{2,5}$,
} Alfred P. Born ${ }^{1}$ and William F.C. Baaré ${ }^{2}$

BACKGROUND: Perinatal exposure to glucocorticoids and elevated endogenous glucocorticoid levels during childhood can have detrimental effects on the developing brain. Here, we examined the impact of glucocorticoid treatment during childhood on brain volumes.

METHODS: A total of 30 children and adolescents with rheumatic or nephrotic disease previously treated with glucocorticoids and 30 controls matched on age, sex, and parent education underwent magnetic resonance imaging (MRI) of the brain. Total cortical gray and white matter, brain, intracranial volume, and total cortical thickness and surface area were derived from MRI scans.

RESULTS: Patients had significantly smaller gray and white matter and total brain volumes relative to healthy controls. Brain volume differences disappeared when accounting for intracranial volume, as patients had relatively smaller intracranial volumes. Group differences were mainly driven by the children with rheumatic disease. Total cortical thickness and cortical surface area did not significantly differ between groups. We found no significant associations between glucocorticoid-treatment variables and volumetric measures. CONCLUSION: Observed smaller total brain, cortical gray, and white matter volumes in children and adolescents previously treated with glucocorticoids compared with that in healthy controls may reflect both developmental and degenerative processes. Prospective longitudinal studies are warranted to clarify whether findings are related to treatment or disease.

G ucocorticoids are widely used in the treatment of pediatric diseases. In the brain, glucocorticoids target the mineralocorticoid and glucocorticoid receptors, which are highly abundant in limbic structures, but are also widely distributed in cortical gray matter and cerebral white matter (1). Treatment with glucocorticoids in the perinatal period has been related to adverse cerebral findings, such as cerebral palsy, lower brain growth, and reduced cortical surface area $(2,3)$. Smaller brain volumes have been reported in animals exposed to exogenous glucocorticoid during fetal life, as well as in children with high endogenous glucocorticoid levels (4). Moreover, prenatal glucocorticoid exposure has been associated with thinner cortex in preadolescents (5). The impact of glucocorticoid treatment on the brain may be influenced by treatment duration, glucocorticoid dose, and the stage of brain development at exposure (6).

The potential harmful mechanisms on neurons and glial cells of excessive levels of glucocorticoids have been widely studied in both animals and humans and include, e.g., inhibition of glucose uptake, decreased cell proliferation, dendritic atrophy, disturbance of cell cycle of the oligodendrocytes, and changes in the cytoskeleton of the neurons (3). In comparison with endogenous glucocorticoids, the exogenous glucocorticoids have different affinities for the glucocorticoid receptors in the brain $(7,8)$. This imbalance in receptor occupancies is believed to affect the synaptic strength and plasticity, cause neuron degeneration, and underlie the cognitive and behavioral side effects that can occur during glucocorticoid treatment (7-10). Glucocorticoids are prescribed in a number of diseases with onset during childhood; however, their potential long-term impact on brain morphology is largely unknown (3). The brain undergoes rapid development in the first years of life and continues to mature during childhood and adolescence and even into early adulthood (11-13). Brain development is regional heterogeneous, with higher associative cortical regions maturing later than primary sensory and motor areas $(11,12,14,15)$. Gray matter volume peaks in early adolescence after which it steadily decreases, whereas white matter volume continues to increase until 40 years of age $(14,16,17)$. Cortical volume, however, is determined by thickness and surface area. Apparent cortical thickness steadily decreases across the entire cortex starting around the age of 3-4 years, whereas cortical surface area increases in a region-specific manner until early adolescence $(16,18,19)$. Although the exact biological mechanisms underlying observed maturational changes are not fully understood, both synaptic pruning and cortical myelination are thought to play a role $(16,20)$. 


\section{Brain volumes and glucocorticoid treatment $\quad$ Articles}

Girls may peak earlier in regional cortical volumes than boys (17), potentially under influence by an earlier onset of puberty. However, these differences have not consistently been found and might be rooted in how sex is modeled statistically (13). The continuing maturation of the brain throughout childhood and adolescence makes it particular vulnerable to both intrinsic and extrinsic disturbances during these periods of life.

The present study aimed to investigate the possible longterm effects of glucocorticoid treatment during childhood on global brain measures. Structural magnetic resonance imaging (MRI) was performed on children and adolescents previously treated with glucocorticoids during childhood for either nephrotic syndrome or rheumatic disease and on their matched healthy controls. By including two different diseases, we aimed to control for potential disease-related effects. Patients and controls were compared on measures of total brain volume, cortical gray matter volume, and white matter volume. Secondly, cortical thickness, surface area, ventricle volume, and intracranial volume were evaluated. Finally, we investigated whether brain measures were associated with glucocorticoid-treatment variables.

\section{METHODS}

\section{Participants and Clinical Characteristics}

A total of 30 children and adolescents previously treated with glucocorticoid because of either rheumatic disease or nephrotic syndrome, recruited from outpatient pediatric clinics, and 30 matched healthy controls, recruited from public schools, were included in the study. Patient and control groups were matched on average age, gender, and parent education. The patients were between 7.0 and 16.1 years of age and consisted of 8 boys and 22 girls. The controls were between 7.0 and 15.6 years of age and consisted of 11 boys and 19 girls. The subjects were part of a larger cohort, which has been described in detail elsewhere (21). Of the initial thirty-eight included patients (21), seven did not complete MRI scanning and one was excluded because of poor image quality due to motion artifacts. Thus, 30 patients with good-quality $\mathrm{T}_{1}$ weighted scans were included in the current study. Of the initially forty-two included healthy controls, five participants did not complete scanning and one control was excluded because of motion artifacts. Thus, good-quality scans were available for 36 healthy controls. To ensure that group averages for included patients were matched to controls on age, gender, and parent education, we excluded five boys and one girl with a mean age of 11.8 years and mean parental education of 17.1 years. Exclusion was done blinded to the MRI data and before any processing of MRI images or statistical analyses. There were no significant differences in age, gender, or parental education between the initial included subjects and the subjects with good-quality MRI scan (all $P$ values $\geqslant 0.30$ ).

Only children and adolescents without neurological or psychiatric diseases or preterm birth were included. Controls were required to be healthy and without previous systemic glucocorticoid treatment. Only patients without current glucocorticoid treatment at the time of the study were included. The study was ethically approved by the Scientific Ethical Committee, Capital Region, Denmark (H-KF-01$131 / 03$ and addendum of June 2009). Written informed consent was obtained from parents of all participants.

\section{Clinical Characteristics}

Patients' medical charts were reviewed and diagnosis and glucocorticoid treatment data were collected. The primary treatment variable was cumulative prednisolone equivalent glucocorticoid dose $(\mathrm{mg} / \mathrm{kg})$.
In addition, median daily dose ( $\mathrm{mg} / \mathrm{kg} /$ day), median age during treatment, and time since treatment (i.e., time elapsed from treatment termination to assessment) were registered. Glucocorticoids were administered orally. However, five patients (one with nephrotic syndrome and four with rheumatic disease) received intravenous glucocorticoid high-dose therapy (pulse therapy) in addition to oral glucocorticoid treatment, which constituted between 36 and $64 \%$ of their cumulative dose.

\section{Background Variables}

The assessment of background and cognitive variables are described in detail elsewhere (21). Height, weight, head circumference, and pubertal development (Tanner staging) were measured. Current and previous physical activity, stressful life events, and behavioral problems were assessed by questionnaires.

\section{Image Acquisition}

The participants were scanned in a 3-Tesla Siemens Magnetom TRIO MR scanner (Siemens, Erlangen, Germany) using an eightchannel head coil (Invivo, Gainesville, FL). We acquired highresolution 3D $\mathrm{T}_{1}$-weighted Magnetization Prepared Rapid Gradient Echo images of the whole brain: repetition time $(\mathrm{TR})=1,550 \mathrm{~ms}$, echo time $(\mathrm{TE})=3.04 \mathrm{~ms}$, matrix $256 \times 256,192$ sagittal slices, no gap, $1 \mathrm{~mm}^{3}$ isotropic voxels.

\section{Image Processing}

Images were processed with FreeSurfer v 5.1.0 (http://surfer.nmr. mgh.harvard.edu). For each subject, we obtained two T1-weighted scans, and the scan of best quality was used for further analysis. A neuroradiologist evaluated acquired scans and all scans were deemed without clinical pathology. The images were processed with FreeSurfer v5.1.0 (http://surfer.nmr.mgh.harvard.edu). The structural processing pipeline in FreeSurfer includes correction of intensity variations due to field in-homogeneities, removal of extracerebral voxels (skull strip), brain tissue classification, and tessellation and deformation producing smooth representations of white matter and pial surfaces $(22,23)$. Generated white matter and pial surfaces were inspected for accuracy and manual editing, and subsequent rerunning of the surface extraction algorithm was performed by a medical doctor (S.K.H.) blinded to subject identity, using standard FreeSurfer procedures. Editing included adjustment of the white matter mask and removing non-brain tissue. Surface-based registration implemented in FreeSurfer allowed for high-accuracy alignment of the cortex in children without introducing age-related bias (24).

\section{Brain Measures}

We measured total brain volume, cortical gray matter volume, white matter volume, cortical thickness, cortical surface area, estimated total intracranial volume, and ventricle volume.

Total brain volume includes supra- and infratentorial volumes of gray and white matter, excluding ventricles, brainstem, and choroid plexus. Total cortical and white matter volume are based on estimated surfaces and not on voxel counts to minimize partial volume effects. Cortical gray matter volume is the volume between the pial and the white matter surface, excluding non-cortical tissue (e.g., hippocampus). Cortical gray matter volume is calculated as the product of cortical thickness and surface area. White mater volume is the volume inside the white matter surface, excluding anything that is not white matter, e.g., ventricles and subcortical gray matter structures. Cortical thickness is measured with subvoxel accuracy as the distance between the pial surface and the white matter surface at each vertex. Surface area is calculated at each vertex as the average area of tessellated triangles surrounding the vertex $(22,23,25)$. Estimated total intracranial volume is estimated using the scaling factor generated in the process of normalizing the T1-weighted image to a template and has been validated against manual delineation of intracranial volume (26). Ventricle volume was calculated as the sum of the lateral, inferior lateral, third, and fourth ventricle volumes $(22,23)$. 


\section{Articles Holm et al.}

\section{Statistical Analyses}

SPSS 20 was used for the statistical analyses (SPSS, IBM Corp., Armonk, NY). $P$ values $\leqslant 0.05$ were considered statistically significant.

Demographic and background variables. A $\chi^{2}$-test tested for possible gender differences. The Shapiro-Wilk test was used to test whether continuous variable distributions deviated from a normal distribution. Independent samples $t$-tests tested for group differences in normally distributed variables, including age and parent education. Group differences in the background, behavioral, and cognitive variables, height, weight, head circumference, verbal comprehension index (VCI), perceptual organization index, Tanner stage, physical activity, stressful life events, behavioral problems, and pattern recognition memory were evaluated using multiple linear regression with age and gender as control variables. The lifetime stressful life event questionnaire includes two statements regarding somatic disease (i.e., "my child had a medical diagnosis" and "my child was severely ill or injured"), which patients and controls are expected to differ on. Therefore, we report stressful life events with and without these disease-related questions. Within patients, Mann-Whitney $U$-tests tested for potential gender- and disease-group differences in treatment variables.

Brain measures. Total brain volume was the primary outcome variable of interest. In case of a significant group (patients vs. controls) effect, we tested for group differences in cortical gray and white matter volume. In addition, we tested for group differences in cortical thickness, surface area, and intracranial and ventricle volume.

Group differences in brain measures were tested with multiple linear regression models controlling for age and gender. Although age and gender were not statistically significantly different between patients and controls, this does not exclude possible inter-individual age and sex effects. As age and gender are the main predictors of structural brain changes that typically occur in the investigated age range, we included both age and sex in all models to adjust for any residual confounding. Furthermore, we tested the anatomical specificity by additionally adjusting for global measures, i.e., estimated total intracranial volume or total brain volume. Estimated total intracranial volume was used to correct total brain volume. Total brain volume was used to correct cortical gray matter volume, white matter volume, and ventricle volume. Finally, we tested for possible group-by-age and group-by-gender interactions, controlling for group, age, gender, and the global measure (see above).

Planned follow-up analyses in previously treated patients were performed for those brain measures on which patients and controls significantly differed. Firstly, we tested for associations with cumulative glucocorticoid dose, median daily dose, treatment duration, median age during treatment, or time since treatment, controlling for age, gender, and global measure. Secondly, we performed repeated analyses while excluding patients who had received pulse therapy. Thirdly, we investigated whether any observed group differences in brain measures remained when controlling for height or lifetime stressful life events without the two disease-related questions. The reason for these analyses was rooted in our observation (see Results section) that patients and controls differed in height and weight. We did not perform any follow-up analyses with weight since weight was highly correlated with height $(r=0.85)$, and we regard height to be a better measure of skeletal and general growth. Finally, we explored whether any observed group difference was present in both disease groups by, respectively, comparing each disease group to controls, controlling for age and gender.

In line with what we observed previously in a larger sample (21), children and adolescents exposed to glucocorticoids had lower verbal intellectual abilities as compared with controls (see under Results). Therefore, we investigated the relationship between inter-individual variation in VCI and brain measures that significantly differed between patients and controls. Brain measures were added as an additional covariate in the main models predicting VCI scores.
We provide an effect-size map of the group differences in cortical gray matter volume to give the reader an impression of the anatomical distribution of group differences across a range of $P$ values uncorrected for multiple comparisons. This effect-size map may be used for comparison with findings of future studies as well as with hypothesis generation. To generate the effect-size map, we performed group comparisons at each vertex of the extracted cortical surfaces using QDEC (Query, Design, Estimate, and Contrast) within FreeSurfer, while controlling for age and gender. In the resulting effect-size map, differences are visualized using blue colors to indicate regions, where patients had lower cortical gray matter volume relative to controls, and red colors to indicate regions, where patients had higher cortical gray matter volume relative to controls.

\section{RESULTS}

\section{Missing Data}

Data were missing for current physical activity (one control and two patients with rheumatic disease), previous physical activity (two patients with rheumatic disease), head circumference (one patient), child-evaluated stressful life events (one patient with rheumatic disease), and lifetime stressful life events (one control and one patient with rheumatic disease). Pubertal development was evaluated using Tanner stages in all participants. Eight patients and four controls, uncomfortable with physical inspection and palpation, filled out a selfestimated pubertal development scheme.

\section{Clinical Characteristics}

The study included 11 patients with nephrotic syndrome and 19 with rheumatic disease. The group of children with nephrotic syndrome included idiopathic nephrotic syndrome $(n=5)$, nephrotic syndrome associated with Henoch Schönleins pupura $(n=4)$, and nephrotic syndrome associated with glomerulonephritis $(n=2)$. The group of children with rheumatic disease included systemic juvenile idiopathic arthritis $(n=1)$, polyarticular juvenile idiopathic arthritis $(n=3)$, oligoarticular juvenile idiopathic arthritis $(n=8)$, enthesisits-related juvenile idiopathic arthritis $(n=2)$, juvenile dermatomyositis $(n=3)$, mixed connective tissue disease $(n=1)$, and systemic lupus erythematosus $(n=1)$.

\section{Background Variables, Cognitive, and Behavioral Measures}

Patients did not differ from controls in age, gender, or parent education (Table 1). The same was true when comparing disease groups separately with controls and when comparing each disease group with one another (Table 1). Statistics on the remaining background variables and the behavioral and cognitive measures are presented in Table 2. Patients had significantly lower weight and height in comparison with control. Head circumference, although lower, was not significantly different between groups. In agreement with our findings in the larger cohort (21), patients had significantly lower VCI compared with that in the controls $(t=-4.4$, $P<0.0001)$, whereas there was no difference regarding the perceptual organization index, memory performance, or behavioral problems. Patients and controls did not significantly differ in pubertal development, physical activity, or stressful life events in the preceding year. In accordance with our previous 


\section{Brain volumes and glucocorticoid treatment Articles}

Table 1. Age, gender, and parent education

\begin{tabular}{|c|c|c|c|c|c|}
\hline & & Controls $(n=30)$ & All patients $(n=30)$ & $\begin{array}{l}\text { Rheumatic disease } \\
(n=19)\end{array}$ & $\begin{array}{c}\text { Nephrotic syndrome } \\
(n=11)\end{array}$ \\
\hline \multirow[t]{3}{*}{ Gender boys/girls } & Boys/girls & $11 / 19$ & $8 / 22$ & $3 / 16$ & $5 / 6$ \\
\hline & Test against controls & - & $P=0.41$ & $P=0.12$ & $P=0.61$ \\
\hline & $\begin{array}{l}\text { Test between disease } \\
\text { groups }\end{array}$ & - & - & & \\
\hline \multirow[t]{3}{*}{ Age (years) } & Mean (SD) & $11.9(2.4)$ & $12.5(2.2)$ & $12.4(2.3)$ & $12.6(2.2)$ \\
\hline & Test against controls & - & $\mathrm{t}=-1.0 ; P=0.33$ & $t=-0.9 ; P=0.42$ & $t=-0.8 ; P=0.42$ \\
\hline & $\begin{array}{l}\text { Test between disease } \\
\text { groups }\end{array}$ & - & - & & \\
\hline \multirow[t]{2}{*}{ Parent education (years) } & Mean (SD) & $14.5(1.6)$ & $14.0(2.0)$ & $13.8(1.5)$ & $14.25(2.7)$ \\
\hline & Range & $11.0-16.5$ & $9.0-17.0$ & $11.5-17.0$ & $9.0-17.0$ \\
\hline
\end{tabular}

$x^{2}$-tests were used to test for gender differences. Possible group differences in age and parent education were evaluated by independent samples $t$-tests.

Table 2. Background, cognitive, and behavioral measures

\begin{tabular}{|c|c|c|c|}
\hline & Controls & Patients & Test statistics \\
\hline Height, $\mathrm{cm}$ (mean $\pm \mathrm{SD})$ & $155.5 \pm 16.0$ & $153.4 \pm 13.0$ & $t=-3.4 ; P=0.0013$ \\
\hline Weight, kg (mean $\pm S D)$ & $47.6 \pm 16.7$ & $44.5 \pm 11.9$ & $t=-2.4 ; P=0.018$ \\
\hline Tanner (median (range)) & $1.5(1-5)$ & $2.3(1-5)$ & $t=-1.7 ; P=0.09$ \\
\hline SLE child last year (median (range)) & $0.05(0-0.21)$ & $0.05(0-0.18)$ & $t=-0.5 ; P=0.60$ \\
\hline SLE lifetime without disease-related questions (median (range)) & $0.07(0-0.19)$ & $0.06(0-0.24)$ & $t=1.3 ; P=0.18$ \\
\hline Current physical activity, h/week (median (range)) & $9.5(2.0-17.5)$ & $9.6(3.3-24.7)$ & $t=0.2 ; P=0.87$ \\
\hline Previous physical activity, h/lived years (median (range)) & $47.5(1.0-145)$ & $41.2(0.5-180.0)$ & $t=0.1 ; P=0.90$ \\
\hline Total problem behavior (median (range)) & $10(1-42)$ & $13(1-42)$ & $t=1.0 ; P=0.32$ \\
\hline Perceptional organization index (median (range)) & $100(60-124)$ & $92(50-124)$ & $t=-1.2 ; P=0.21$ \\
\hline Verbal comprehension index (mean $\pm S D$ ) & $112.5 \pm 15.6$ & $95.6 \pm 16.6$ & $t=-4.4 ; P<0.0001$ \\
\hline
\end{tabular}

SLE, stressful life events.

For variables with normal distribution mean and SD are supplied, for the remaining variables median and range are reported. Independent samples $t$-tests tested for group differences in age and parent education. Multiple linear regression, controlling for age and gender, tested for group differences in height, weight, head circumference, Tanner stage, stressful life events, physical activity, behavioral problems, pattern recognition memory, perceptual organization index, and verbal comprehension index.

findings in a larger cohort (21), the amount of stressful life events during the child's entire lifetime was higher in patients compared with that in controls; however, after exclusion of disease-related questions, this difference was no longer present.

Treatment variables are presented in Table 3. Children with nephrotic syndrome had received significantly higher cumulative and daily glucocorticoid doses than children with rheumatic disease. Patient groups did not differ on other treatment variables (Table 3). Furthermore, relative to girls, boys had received significantly higher daily doses and more time had elapsed since termination of the glucocorticoid treatment (Table 3).

\section{Brain Measures}

The mean values for brain measures for all groups are presented in Table 4. Results from multiple regression models are presented in Table 5.

Total brain volume. Patients had significantly smaller total brain volume as compared with that of controls, controlling for 


\section{Articles Holm et al.}

Table 3. Glucocorticoid treatment variables

\begin{tabular}{|c|c|c|c|c|c|c|c|}
\hline Treatment variables; median (range) & All patients & Nephrotic syndrome & Rheumatic disease & $P^{a}$ & Boys & Girls & $P^{\mathrm{b}}$ \\
\hline Age during treatment (years) & $7.0(1.9-12.3)$ & $7.7(4.4-10.5)$ & $6.7(1.9-12.3)$ & 0.40 & $7.0(5.1-9.8)$ & $7.1(1.9-12.3)$ & 0.99 \\
\hline Cumulative dose $(\mathrm{mg} / \mathrm{kg})$ & $158(21-580)$ & $261(119-514)$ & $184(21-580)$ & 0.03 & $237(74-514)$ & $147(21-580)$ & 0.50 \\
\hline Median daily dose (mg/kg/day) & $0.2(0.1-2.1)$ & $0.6(0.1-2.1)$ & $0.3(0.1-1.1)$ & 0.03 & $0.6(0.1-2.1)$ & $0.2(0.1-1.1)$ & 0.05 \\
\hline Treatment duration (years) & $1.0(0.1-5.7)$ & $1.1(0.3-3.9)$ & $1.4(0.1-5.7)$ & 0.50 & $1.1(0.5-1.8)$ & $0.8(0.1-5.7)$ & 0.95 \\
\hline Time since treatment (years) & $4.0(0.2-8.9)$ & $4.2(0.6-8.9)$ & $3.5(0.2-6.8)$ & 0.55 & $5.0(2.4-8.9)$ & $3.4(0.2-6.8)$ & 0.02 \\
\hline
\end{tabular}

Treatment variables are given as median (range) for all patients, for nephrotic and rheumatic disease groups, and boys and girls separately. Doses are given as prednisolone equivalents and include pulse therapy. $P$ values supplied are from Mann-Whitney U-tests.

a Nephrotic syndrome vs. rheumatic disease.

boys vs. girls.

$P$ values $\leqslant 0.05$ are presented in bold italic font.

Table 4. Brain measures

\begin{tabular}{|c|c|c|c|c|}
\hline Brain measures; mean (SD) & Controls $(N=30)$ & All patients $(N=30)$ & Nephrotic syndrome $(N=11)$ & Rheumatic disease $(N=19)$ \\
\hline Total brain volume $\left(\mathrm{cm}^{3}\right)$ & $1,264(108)$ & 1,201 (104) & $1,230(88)$ & 1,185 (112) \\
\hline Cortical gray matter volume $\left(\mathrm{cm}^{3}\right)$ & $613(48)$ & $581(47)$ & $593(48)$ & $574(46)$ \\
\hline Cortical surface area $\left(\mathrm{cm}^{2}\right)$ & $197(16)$ & $191(15)$ & $193(12)$ & $190(17)$ \\
\hline White matter volume $\left(\mathrm{cm}^{3}\right)$ & $473(56)$ & $444(55)$ & $454(40)$ & $439(62)$ \\
\hline
\end{tabular}

Brain measures given as mean (SD).

Table 5. Results from multiple regression analyses of brain measures

\begin{tabular}{|c|c|c|c|c|c|c|}
\hline \multirow[t]{2}{*}{ Model } & \multirow[t]{2}{*}{ Brain measure } & \multicolumn{2}{|c|}{ Group } & \multirow{2}{*}{$\begin{array}{l}\text { Age } \\
\beta ; P\end{array}$} & \multirow{2}{*}{$\begin{array}{c}\text { Gender } \\
\beta ; P\end{array}$} & \multirow{2}{*}{$\begin{array}{c}\text { Global measure } \\
\beta ; P\end{array}$} \\
\hline & & AMD $(95 \% \mathrm{Cl})$ & $\beta ; P$ & & & \\
\hline 1 & Total brain volume $\left(\mathrm{cm}^{3}\right)$ & $-60(-114 ;-5)$ & $\beta=-0.27 ; P=0.03$ & $\beta=0.01 ; P=0.51$ & $\beta=-0.25 ; P=\mathbf{0 . 0 5}$ & - \\
\hline 2 & Total brain volume $\left(\mathrm{cm}^{3}\right)$ & $-24(-54 ; 6)$ & $\beta=-0.11 ; P=0.12$ & $\beta=-0.22 ; P=\mathbf{0 . 0 0 4}$ & $\beta=0.04 ; P=0.61$ & $\beta=0.88 ; P<\mathbf{0 . 0 0 1}$ \\
\hline 1 & Cortical gray matter volume $\left(\mathrm{cm}^{3}\right)$ & $-28(-53 ;-4)$ & $\beta=-0.29 ; P=0.03$ & $\beta=-0.15 ; P=0.23$ & $\beta=-0.21 ; P=0.10$ & - \\
\hline 2 & Cortical gray matter volume $\left(\mathrm{cm}^{3}\right)$ & $-4(-15 ; 72)$ & $\beta=-0.04 ; P=0.50$ & $\beta=-0.23 ; P<0.001$ & $\beta=0.21 ; P=0.71$ & $\beta=0.91 ; P<\mathbf{0 . 0 0 1}$ \\
\hline 1 & Cortical thickness (mm) & $-0.05(-0.10 ; 0.01)$ & $\beta=-0.22 ; P=0.08$ & $\beta=-0.28 ; P=0.03$ & $\beta=0.01 ; P=0.45$ & - \\
\hline 1 & Cortical surface area $\left(\mathrm{cm}^{2}\right)$ & $-6(-14 ; 3)$ & $\beta=-0.18 ; P=0.17$ & $\beta=-0.01 ; P=0.92$ & $\beta=-0.29 ; P=0.03$ & - \\
\hline 1 & White matter volume $\left(\mathrm{cm}^{3}\right)$ & $-29(-57 ;-15)$ & $\beta=-0.26 ; P=0.04$ & $\beta=0.24 ; P=0.06$ & $\beta=-0.23 ; P=0.07$ & - \\
\hline 2 & White matter volume $\left(\mathrm{cm}^{3}\right)$ & $-1(-13 ; 10)$ & $\beta=-0.01 ; P=0.83$ & $\beta=0.16 ; P=0.002$ & $\beta<0.01 ; P=0.99$ & $\beta=0.90 ; P<0.001$ \\
\hline 1 & Estimated total intracranial volume & $-55(-126 ; 15)$ & $\beta=-0.19 ; P=0.12$ & $\beta=0.34 ; P=0.007$ & $\beta=-0.24 ; P=0.05$ & - \\
\hline 1 & Ventricle volume $\left(\mathrm{cm}^{3}\right)$ & $-0.4(-2.1 ; 1.5)$ & $\beta=-0.05 ; P=0.66$ & $\beta=-0.34 ; P=0.02$ & $\beta=-0.34 ; P=0.006$ & - \\
\hline 2 & Ventricle volume $\left(\mathrm{cm}^{3}\right)$ & $-0.1(2.0 ; 1.8)$ & $\beta=-0.01 ; P=0.91$ & $\beta=0.23 ; P=0.08$ & $\beta=-0.29 ; P=0.02$ & $\beta=0.21 ; P=0.12$ \\
\hline
\end{tabular}

Cl, confidence interval.

Group: compares patients vs. controls. Model 1: age and gender were control variables. Model 2: age, gender, and global measure were control variables. Global measure: estimated intracranial volume for total brain and ventricle volume, and total brain volume for cortical gray and white matter volume. Negative values reflect smaller measures in patients or girls. AMD is the adjusted mean difference in brain volumes between patients and control.

$P$ values $\leqslant 0.05$ are presented in bold italic font.

age and gender (Table 5). When controlling for estimated total intracranial volume this difference was no longer significant $(\beta=-0.11, P=0.12)$. There was no significant group-by-age $(\beta=0.38, P=0.07)$ or group-by-gender $(\beta=-0.02, P=0.81)$ interaction. In patients, we found no significant associations between total brain volume and any of the treatment variables ( $P$ value range $=0.21-0.49)$. Follow-up analysis adding stressful life events without the two disease-related questions (SLE-LT-) appeared not to have a large impact on the group difference (group: $\beta=-0.24, P=0.07$; age: $\beta=0.08, P=0.57$; gender: $\beta=-0.25, P=0.09$; SLE-LT-: $\beta=-0.07, P=0.57$ ). Adding height as a covariate revealed that the group difference in total brain volume was no longer significant (group: $\beta=-0.19$, $P=0.16$; age: $\beta=-0.30, P=0.31$; gender: $\beta=-0.23, P=0.06$; height: $\beta=0.42, P=0.16$ ). Collinearity diagnostics revealing tolerances of 0.17 and variance inflation factors of 5.8 for age 


\section{Brain volumes and glucocorticoid treatment Articles}
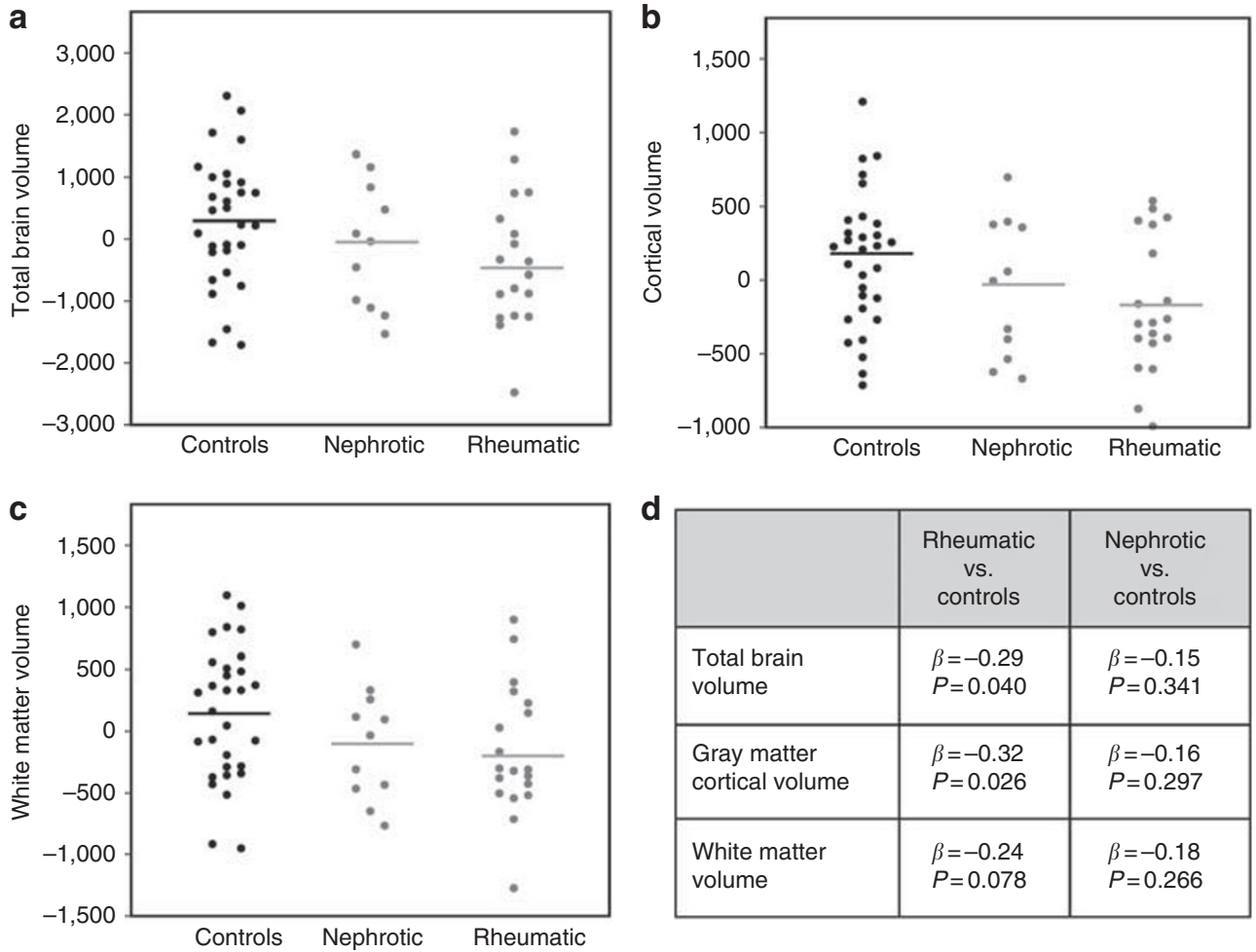

Figure 1. Partial regression plots of differences between patients and controls. Total brain volume (a), cortical gray matter volume (b), and white matter volume (c). Horizontal lines represent means for controls and the two disease groups. The partial regression plots render values to be arbitrary. Results from comparing controls with the separate disease groups are presented in (d). Results and plots are controlled for age and gender.

and height suggest collinearity between these two factors). When comparing the disease groups separately with the healthy controls, total brain volume was smaller in children with rheumatic disease, but not in children with nephrotic syndrome controlling for age and gender (Figure 1a,d). Excluding individuals who received pulse therapy did not affect these results.

Individual VCI scores showed a positive linear relationship with total brain volume $(\beta=0.46, P=0.046)$, when simultaneously modeling group: $\beta=-0.45, P<0.001$; age: $\beta=0.33$, $P=0.01$; gender: $\beta=0.05, P=0.71$; and estimated total intracranial volume: $\beta=-0.40, P=0.10$.

Cortical gray matter volume. Cortical gray matter volume was significantly smaller in patients compared with that in the controls, controlling for age and gender (Table 5). This group difference became nonsignificant when adjusting for total brain volume $(\beta=-0.04, P=0.50)$. There was no significant groupby-age $(\beta=-0.27, P=0.11)$ or group-by-gender $(\beta=-0.07$, $P=0.19$ ) interaction effect. We did not observe any associations between cortical gray matter volume and treatment variables $(P$ value range $=0.63-0.97)$. Follow-up analyses adding SLE-LT- as a covariate appeared not to have a large impact on the group difference (group: $\beta=-0.25$, $P=0.06$; age: $\beta=-0.17, P=0.20$; gender: $\beta=-0.17, P=0.19$; SLE-LT-: $\beta=-0.14, P=0.28$ ). Adding height as a covariate revealed that the group difference in cortical gray matter volume became statistically insignificant $(\beta=-0.25, P=0.08$; age: $\beta=-0.33, P=0.28$; gender: $\beta=-0.20, P=0.12$; height: $\beta=0.19$,
$P=0.52$ ). Age and height were collinear (see under "total brain volume"). Separate analyses of the two disease groups showed smaller cortical gray matter volume in children with rheumatic disease, whereas children with nephrotic syndrome did not differ significantly from controls controlling for age and gender (Figure 1b,d). Excluding individuals, who received pulse therapy, did not affect these results. When including cortical gray matter volume in the model as a predictor of VCI, VCI remained significantly lower in patients compared with that in controls, and cortical gray matter volume and VCI were negatively associated (group: $\beta=-0.49, P<0.001$; cortical gray matter volume: $\beta=-0.71, P=0.01$; age: $\beta=0.06, P=0.63$; gender: $\beta=0.08, \quad P=0.51$; total brain volume: $\beta=0.79$, $P=0.007)$.

The surface-based effect-size map of group differences in cortical gray matter volume, controlling for age and gender, showed widespread cortical areas with smaller volume in patients relative to controls with no apparent anatomical predilection (Supplementary Figure S1 online).

White matter volume. Patients had significantly smaller white matter volume compared with the volume in controls, adjusted for age and gender (Table 5). This group difference became nonsignificant when adjusting for total brain volume. There was a significant group-by-age interaction on white matter volume $(\beta=0.33, P=0.03)$, with patients showing a positive association between age and white matter volume ( $\beta=0.55, P=0.002)$, although age seemed not to be associated 


\section{Articles $\mid$ Holm et al.}

with white matter volume in controls $(\beta=-0.02, P=0.89)$. Dividing the group in two by the median age showed that younger patients had smaller WM volumes than younger controls $(\beta=-0.54, P=0.002)$, whereas older patients and controls did not differ from each other $(\beta=-0.28, P=0.89)$. There was no significant group-by-gender interaction $(\beta=0.04$, $P=0.45)$. We did not observe any associations between white matter volume and treatment variables $(P$ values range $=0.30$ 0.75). Follow-up analyses adding SLE-LT- appeared not to have a large impact on the group difference (group: $\beta=-0.23$, $P=0.08$; age: $\beta=0.24, P=0.07$; gender: $\beta=-0.23, P=0.08$; SLE-LT-: $\beta=-0.04, P=0.76)$. Adding height as a covariate revealed that the group difference in white matter volume was no longer significant (group: $\beta=-0.16, P=0.24$; age: $\beta=-0.25, \quad P=0.38$; gender: $\beta=-0.21, \quad P=0.09$; height: $\beta=0.54, P=0.06$ ). Age and height were collinear (as under "total brain volume"). Analyses of the disease groups separately did not reveal any significant differences with controls, although white matter volume differences appeared to be somewhat more pronounced in children with rheumatic disease than in children with nephrotic syndrome (Figure 1c, d). The observed differences between patients and controls did not change when excluding individuals, who received pulse therapy.

Including white matter volume as a predictor for VCI, VCI remained significantly lower in patients, and white matter volume was positively related to VCI (Group: $\beta=-0.46$, $P<0.001$; white matter volume: $\beta=0.64, P=0.05$; age: $\beta=0.18, P=0.13$ : gender: $\beta=0.06, P=0.60$; total brain volume; $\beta=-0.44, P=0.16)$.

Cortical thickness and surface area. Cortical thickness did not differ between patients and healthy controls when controlling for age and gender (Table 5). There was no significant group-by-age $(\beta=-0.64, P=0.10)$ or group-bygender $(\beta=-0.16, P=0.23)$ interaction.

Cortical surface area did not significantly differ between patients and controls (Table 5). There was no significant group-by-age $(\beta=0.7, P=0.09)$ or group-by-gender interaction $(\beta=0.07, P=0.60)$.

Estimated total intracranial volume. Patients did not have significantly lower intracranial volumes than controls (estimated total intracranial volume: $\beta=-0.19, P=0.12$, Table 5). There was also no significant group-by-age $(\beta=0.19, P=0.61)$ or group-by-gender $(\beta=0.09, P=0.49)$ interaction effects.

Ventricle volume. Ventricle volume was not significantly different between controls and patients, without or with controlling for estimated total intracranial volume (Table 5). There was no group-by-age $(\beta=0.09, P=0.82)$ or group-bygender interaction $(\beta=-0.16, P=0.21)$.

\section{DISCUSSION}

To the best of our knowledge, this is the first study to investigate associations between glucocorticoid treatment during childhood because of extracerebral diseases and brain volumes later in childhood and adolescence. We found that children previously treated with glucocorticoids for rheumatic disease or nephrotic syndrome had significantly smaller total brain volumes as compared with the controls, including both lower cortical gray and lower white matter volumes in patients. The difference in total brain volume did not reach statistical significance when accounting for intracranial volume, suggesting that smaller brains were confined to smaller intracranial cavities and were not likely the result of brain atrophy per se. If the latter were the case, we would have expected to find that group difference in total brain volume would have become more pronounced, e.g., smaller brains in equal-sized intracranial cavities. Because brain growth is the driving force in shaping the intracranial cavity, the observed smaller brain volumes in patients may potentially be due to disease or treatment effects on brain development. Findings of lower height and weight in patients compared with that in controls suggest a general physical growth restriction in patients. Although group differences in total brain, cortical gray, and white matter volume were no longer significant when controlling for height, these results are not easily interpreted, given the observed collinearity between age and height. Studies of body growth and brain development suggest that brain size is not determined by body size (27). Furthermore, a recent study of 14,819 children under 7 years of age found that after the age of 2 head growth seemed to progress independently of skeletal growth and body size (28). Therefore, different mechanisms may underlie the observed restricted brain and body development.

A clear appraisal of our main findings is hampered by the lack of previous imaging studies investigating the long-term impact of glucocorticoid treatment during childhood because of extracerebral diseases. Nevertheless, smaller total brain volume has been associated with high endogenous glucocorticoid levels in children and adults $(29,30)$. Furthermore, apparent brain atrophy has been found in children with epilepsy treated with glucocorticoids or adrenocorticotrophic hormone (31-33). Yano et al. also included a group of glucocorticoid-treated children with nephrotic syndrome in whom reversible brain atrophy was detected using computed tomography. However, in these studies the causality is unclear as the glucocorticoid treatment is used to treat a neurological disease, which in itself might affect the brain volume. Furthermore, children born extremely preterm ( $<28$ weeks) and exposed to glucocorticoids in the neonatal period seem to have reduced total brain volume and lower white matter volume in adolescence, whereas cortical gray matter volume appeared to be unaffected (34). In summary, although preliminary, our results seem to be in line with findings of studies investigating subjects with elevated endogenous glucocorticoid levels, children receiving glucocorticoid treatment for diseases affecting the brain, and studies of perinatal exposure to glucocorticoids.

In contrast to our expectations, we found no associations between glucocorticoid-treatment variables and any of the brain measures. Moreover, follow-up analyses suggested that 


\section{Brain volumes and glucocorticoid treatment Articles}

our finding of smaller brain and cortical gray and white matter volumes in patients were mainly driven by the children with rheumatic disease, suggesting that our findings may be related to rheumatic disease and not glucocorticoid treatment per se. Although speculative, the notion that the children with rheumatic disease also received lower glucocorticoid doses than the children with nephrotic syndrome underscores such an interpretation. The majority of children with rheumatic disease were diagnosed with juvenile idiopathic arthritis. Both exogenous glucocorticoids and the pro-inflammatory cytokines involved in the pathogenesis of JIA seem to interfere with growth hormone and insulin-like-growth factor $(35,36)$, both of which are known to affect brain growth, myelination, neurogenesis, and cognition $(37,38)$. However, the observations of effects potentially related to rheumatic disease may also be related to clinical characteristics, such as disease severity, impact on daily life, or degree of inflammation. Indeed, although we could not find any studies directly comparing JIA with nephrotic syndrome, a qualitative comparison of symptoms with disease courses suggests that children with rheumatic disease experience longer periods of pain, with a potentially larger impact on daily life, than those children with nephrotic syndrome $(39,40)$. Furthermore, the cross-sectional nature of the study prevents us from examining whether the observed smaller cortical gray and white matter volumes were causally related to glucocorticoid treatment or whether they were a consequence of having rheumatic disease or nephrotic syndrome per se. Our observation that smaller brain volumes in patients appeared mainly to be driven by the children with rheumatic disease seems to point in the latter direction. Nevertheless, given the lower number of patients with nephrotic syndrome, we may have lacked the statistical power to reveal any differences between patients with nephrotic syndrome and healthy controls. Furthermore, because there was a dominance of girls in the group with rheumatic disease, gender-specific mechanisms may also have contributed. Moreover, we had no measure of disease severity, which would have been an interesting clinical measurement, but as the study was retrospective, such an assessment was not found feasible. Further studies are needed to disentangle glucocorticoid treatment effects from disease-specific effects. Given the relative small sample size and associated limited statistical power, it is important to stress that lack of finding an effect does not equal the non-existence of an effect. Furthermore, the study is limited by the nature of the control group, and arguably a group of diseased children not treated with steroids might be a better control group. However, differences in specific disease mechanisms, illness impact and course, and treatment severely hamper identifying a suitable disease control group. Finally, the study design only allows us to describe associations and no causality can be claimed.

In conclusion, observed smaller total brain, cortical gray, and white matter volumes in children and adolescents previously treated with glucocorticoids as compared with that in the healthy controls may reflect both developmental and degenerative processes. The current study findings should be considered preliminary and need replication. Moreover, prospective and longitudinal studies are required to determine whether our findings are related to glucocorticoid treatment or disease-specific effects.

\section{SUPPLEMENTARY MATERIAL}

Supplementary material is linked to the online version of the paper at http://www.nature.com/pr

\section{ACKNOWLEDGMENTS}

Our greatest acknowledgment is directed toward all participating children and their parents. Furthermore, The Danish Research Council for Independent Research, the Lundbeck Foundation, and The Ville Heise Foundation are acknowledged for kindly funding the study.

\section{STATEMENT OF FINANCIAL SUPPORT}

All phases of this study were kindly supported by The Danish Council for Independent Research (grant number: 09-071546), The Lundbeck Foundation (grant number: R48-A4968), and The Ville Heise Foundation.

Disclosure: Financial disclosure: Hartwig Siebner has received honoraria as speaker from Lundbeck A/S, Denmark, Biogen Idec, Denmark, Genzyme, Denmark, and MerckSerono, Denmark, honoraria from Elsevier Publishers, Amsterdam, The Netherlands, and Springer Publishing, Stuttgart, Germany, and travel support from MagVenture, Denmark. The remaining authorsdeclare no conflict of interest.

\section{REFERENCES}

1. Cao-Lei L, Suwansirikul S, Jutavijittum P, et al. Glucocorticoid receptor gene expression and promoter CpG modifications throughout the human brain. J Psychiatr Res 2013;47:1597-607.

2. Doyle LW, Ehrenkranz RA, Halliday HL. Early ( $<8$ days) postnatal corticosteroids for preventing chronic lung disease in preterm infants. Cochrane Database Syst Rev 2014;5:CD001146.

3. Damsted SK, Born AP, Paulson OB, Uldall P. Exogenous glucocorticoids and adverse cerebral effects in children. Eur J Paediatr Neurol 2011;15: 465-77.

4. Salpietro V, Polizzi A, Di RG, et al. Adrenal disorders and the paediatric brain: pathophysiological considerations and clinical implications. Int J Endocrinol 2014;2014:282489.

5. Davis EP, Sandman CA, Buss C, et al. Fetal glucocorticoid exposure is associated with preadolescent brain development. Biol Psychiatry 2013;74: 647-55.

6. Malaeb SN, Stonestreet BS. Steroids and injury to the developing brain: net harm or net benefit? Clin Perinatol 2014;41:191-208.

7. de Kloet ER, Vreugdenhil E, Oitzl MS, Joels M. Brain corticosteroid receptor balance in health and disease. Endocr Rev 1998;19:269-301.

8. de Kloet ER.. From receptor balance to rational glucocorticoid therapy. Endocrinology 2014;155:2754-69.

9. McEwen BS, Angulo J, Cameron H, et al. Paradoxical effects of adrenal steroids on the brain: protection versus degeneration. Biol Psychiatry 1992;31:177-99.

10. McEwen BS. Corticosteroids and hippocampal plasticity. Ann N Y Acad Sci 1994;746:134-42.

11. Tamnes CK, Herting MM, Goddings AL, et al. Development of the cerebral cortex across adolescence: a multisample study of inter-related longitudinal changes in cortical volume, surface area, and thickness. J Neurosci 2017;37:3402-12.

12. Jernigan TL, Baare WF, Stiles J, Madsen KS. Postnatal brain development: structural imaging of dynamic neurodevelopmental processes. Prog Brain Res 2011;189:77-92.

13. Mills KL, Goddings AL, Herting MM, et al. Structural brain development between childhood and adulthood: convergence across four longitudinal samples. Neuroimage 2016;141:273-81. 


\section{Articles | Holm et al.}

14. Coupe P, Catheline G, Lanuza E, Manjon JV. Towards a unified analysis of brain maturation and aging across the entire lifespan: a MRI analysis. Hum Brain Mapp 2017;38:5501-18.

15. Giedd JN, Stockman M, Weddle C, et al. Anatomic magnetic resonance imaging of the developing child and adolescent brain and effects of genetic variation. Neuropsychol Rev 2010;20:349-61.

16. Walhovd KB, Fjell AM, Giedd J, et al. Through thick and thin: a need to reconcile contradictory results on trajectories in human cortical development. Cereb Cortex 2017;27:1472-81.

17. Lenroot RK, Gogtay N, Greenstein DK, et al. Sexual dimorphism of brain developmental trajectories during childhood and adolescence. Neuroimage 2007;36:1065-73.

18. Amlien IK, Fjell AM, Tamnes CK, et al. Organizing principles of human cortical development-thickness and area from 4 to 30 years: insights from comparative primate neuroanatomy. Cereb Cortex 2016;26:257-67.

19. Brown TT, Kuperman JM, Chung Y, et al. Neuroanatomical assessment of biological maturity. Curr Biol 2012;22:1693-8.

20. Paus T. Growth of white matter in the adolescent brain: myelin or axon? Brain Cogn 2010;72:26-35.

21. Holm SK, Vestergaard M, Madsen KS, et al. Children and adolescents previously treated with glucocorticoids display lower verbal intellectual abilities. Acta Paediatr 2015;104:784-91.

22. Dale AM, Fischl B, Sereno MI. Cortical surface-based analysis. I. Segmentation and surface reconstruction. Neuroimage 1999;9:179-94.

23. Fischl B, Sereno MI, Dale AM. Cortical surface-based analysis. II: Inflation, flattening, and a surface-based coordinate system. Neuroimage 1999;9:195-207.

24. Ghosh SS, Kakunoori S, Augustinack J, et al. Evaluating the validity of volume-based and surface-based brain image registration for developmental cognitive neuroscience studies in children 4 to 11 years of age. Neuroimage 2010;53:85-93.

25. Fischl B, Dale AM. Measuring the thickness of the human cerebral cortex from magnetic resonance images. Proc Natl Acad Sci USA 2000;97:11050-5.

26. Buckner RL, Head D, Parker J, et al. A unified approach for morphometric and functional data analysis in young, old, and demented adults using automated atlas-based head size normalization: reliability and validation against manual measurement of total intracranial volume. Neuroimage 2004;23:724-38.
27. Mills KL, Tamnes CK. Methods and considerations for longitudinal structural brain imaging analysis across development. Dev Cogn Neurosci 2014;9:172-90.

28. Scheffler C, Greil H, Hermanussen M. The association between weight, height, and head circumference reconsidered. Pediatr Res 2017;81: $825-30$.

29. Merke DP, Giedd JN, Keil MF, et al. Children experience cognitive decline despite reversal of brain atrophy one year after resolution of Cushing syndrome. J Clin Endocrinol Metab 2005;90:2531-6.

30. Patil CG, Lad SP, Katznelson L, Laws ER Jr. Brain atrophy and cognitive deficits in Cushing's disease. Neurosurg Focus 2007;23:1-4.

31. Ito M, Takao T, Okuno T, Mikawa H. Sequential CT studies of 24 children with infantile spasms on ACTH therapy. Dev Med Child Neurol 1983;25:475-80.

32. Konishi Y, Yasujima M, Kuriyama M, et al. Magnetic resonance imaging in infantile spasms: effects of hormonal therapy. Epilepsia 1992;33: 304-9.

33. Yano E. Apparent cerebral atrophic findings on cranial computed tomography in nephrotic children with steroid therapy and in patients of infantile spasms with ACTH therapy. Kurume Med J 1981;28:63-77.

34. Cheong JL, Burnett AC, Lee KJ, et al. Association between postnatal dexamethasone for treatment of bronchopulmonary dysplasia and brain volumes at adolescence in infants born very preterm. J Pediatr 2014;164: 737-43.

35. Gaspari S, Marcovecchio ML, Breda L, Chiarelli F. Growth in juvenile idiopathic arthritis: the role of inflammation. Clin Exp Rheumatol 2011;29:104-10.

36. Hochberg Z. Mechanisms of steroid impairment of growth. Horm Res 2002;58 (Suppl 1): 33-8.

37. Webb EA, O'Reilly MA, Clayden JD, et al. Effect of growth hormone deficiency on brain structure, motor function and cognition. Brain 2012;135:216-7.

38. Aberg D. Role of the growth hormone/insulin-like growth factor 1 axis in neurogenesis. Endocr Dev 2010;17:63-76.

39. Eddy AA, Symons JM. Nephrotic syndrome in childhood. Lancet 2003;362: 629-39.

40. Ravelli A, Martini A. Juvenile idiopathic arthritis. Lancet 2007;369:767-8. 